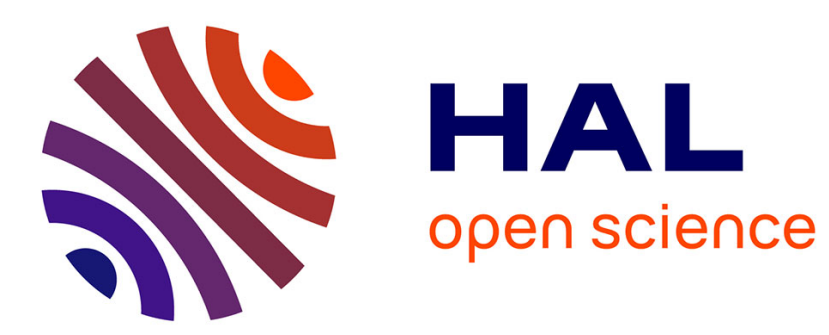

\title{
Majorantes surharmoniques minimales d'une fonction continue
}

Jean Jacques Moreau

\section{To cite this version:}

Jean Jacques Moreau. Majorantes surharmoniques minimales d'une fonction continue. Annales de l'Institut Fourier, 1971, 21 (2), pp.129 - 156. 10.5802/aif.375 . hal-01788488

\section{HAL Id: hal-01788488 \\ https://hal.science/hal-01788488}

Submitted on 9 May 2018

HAL is a multi-disciplinary open access archive for the deposit and dissemination of scientific research documents, whether they are published or not. The documents may come from teaching and research institutions in France or abroad, or from public or private research centers.
L'archive ouverte pluridisciplinaire HAL, est destinée au dépôt et à la diffusion de documents scientifiques de niveau recherche, publiés ou non, émanant des établissements d'enseignement et de recherche français ou étrangers, des laboratoires publics ou privés. 


\section{ANNALES DE L'INSTITUT FOURIER}

\section{JEAN-JACQUES MOREAU}

\section{Majorantes surharmoniques minimales d'une fonction continue}

Annales de l'institut Fourier, tome 21, nº 2 (1971), p. 129-156.

<http://www.numdam.org/item?id=AlF_1971_21_2_129_0>

(C) Annales de l'institut Fourier, 1971, tous droits réservés.

L'accès aux archives de la revue «Annales de l'institut Fourier » (http://annalif.ujf-grenoble.fr/), implique l'accord avec les conditions générales d'utilisation (http://www.numdam.org/legal.php). Toute utilisation commerciale ou impression systématique est constitutive d'une infraction pénale. Toute copie ou impression de ce fichier doit contenir la présente mention de copyright.

\section{Numdam}

Article numérisé dans le cadre du programme Numérisation de documents anciens mathématiques http://www.numdam.org/ 
Ann. Inst. Fourier, Grenoble

21, 2 (1971), 129-156.

\title{
MAJORANTES SURHARMONIQUES MINIMALES D'UNE FONCTION CONTINUE
}

\author{
par Jean-Jacques MOREAU
}

\section{Introduction.}

1.a. Soit $f$ une fonction numérique finie et continue dans un ouvert $\Omega$ de $\mathbf{R}^{n}$. Nous disons qu'une majorante surharmonique de $f$ dans $\Omega$ est minimale (ou, abréviativement, que cette fonction est ( une minimale de $f$ ) si cette majorante surharmonique est harmonique dans l'ensemble (ouvert) où elle diffère de $f$. Cet emploi du mot minimal est justifié par l'introduction d'une certaine relation d'ordre dans l'ensemble des majorantes surharmoniques de $f$.

Ces fonctions sont continues; une minimale d'une minimale de $f$ est une minimale de $f$; il en existe une plus petite que toutes les autres et dont toutes les autres sont des minimales. Cette dernière est tout simplement la plus petite majorante surharmonique de $f$ dont l'existence est connue depuis longtemps (N. Sjöberg [16], M. Brelot [1]).

L'idée dominante de la présente théorie est qu'une analogie très profonde existe entre les propriétés des minimales d'une fonction continue donnée $f$ et les propriétés des fonctions harmoniques $\geqslant 0$ dans l'ouvert $\Omega$; il s'agit en fait d'une généralisation puisque les fonctions harmoniques $\geqslant 0$ sont les minimales de la fonction constante $f=0$. Cette généralisation nous semble d'un type nouveau, car non linéaire: la famille des minimales de $f$ ne forme pas un espace vectoriel, ni même un cône convexe.

Passons en revue quelques analogies typiques qui vont se révéler entre fonctions harmoniques $\geqslant 0$ et minimales d'une fonction donnée $f$. 
Si $u$ et $\varphi$ sont deux minimales de $f$, on verra que la fonction continue $|u-\vartheta|$ est sous-harmonique, donc justiciable $\mathrm{du}$ principe du minimum. Cela servira, notamment, dans l'étude du problème de Dirichlet: étant donnée $u^{*}$, fonction continue définie sur la frontière $\Omega^{*}$ de $\Omega$ (frontière prise relativement à l'espace compactifié $\left.\hat{\mathbf{R}}^{n}\right)$, trouver $u$, fonction continue dans l'adhérence $\hat{\Omega}$ de $\Omega$, minimale de $f$ dans $\Omega$ et admettant $u^{*}$ pour restriction à $\Omega^{*}$. On trouvera que (moyennant une condition de possibilité évidente entre $u^{*}$ et $f$ ) ce problème admet une solution si $\Omega$ est régulier pour le problème de Dirichlet traditionnel. Cette solution est unique; c'est la plus petite fonction s.c.i. dans $\hat{\Omega}$, surharmonique et majorant $f$ dans $\Omega$, majorant $u^{*}$ dans $\Omega^{*}$. La dépendance de cette solution $u$ à l'égard de la donnée $u^{*}$ fournit une situation caractéristique de ce que nous appelons l'analyse unilatérale: pour chaque $x_{0} \in \Omega$, l'application $u^{*} \longmapsto u\left(x_{0}\right)$ est convexe continue (d'une partie) de l'espace de Banach $\mathfrak{C}\left(\Omega^{*}\right)$ dans $\mathbf{R}$; cette fonctionnelle convexe (croissante) joue le rôle que tient la fonctionnelle linéaire positive "mesure harmonique " dans le problème de Dirichlet classique. Naturellement, comme pour le problème de Dirichlet classique, l'adéquation de $u$ à la donnée $u^{*}$ pourrait être entendue en un sens plus faible, ce qui affranchirait de l'hypothèse de régularité de $\Omega$.

Les familles de minimales de $f$ possèdent des propriétés tout à fait semblables à celles des familles de fonctions harmoniques $\geqslant 0$ : Dans l'ensemble des minimales de $f$, toute partie non vide possède un plus grand minorant; toute partie majorée possède un plus petit majorant. L'enveloppe inférieure d'une famille de minimales de $f$ est une fonction continue. Supposons $\Omega$ connexe; l'enveloppe supérieure d'une famille de minimales de $f$ est soit la constante $+\infty$, soit une fonction partout finie et continue. Dans le deuxième cas, la famille en question est uniformément équicontinue sur tout compact $\mathrm{K} \subset \Omega$, au sens de la structure uniforme additive de $\mathbf{R}$; d'une façon générale, l'ensemble des minimales de $f$ est uniformément équicontinu sur tout compact $K \subset \Omega$ au sens de la structure uniforme de $\overline{\mathbf{R}}$. On tire de là que si une suite généralisée de minimales de $f$ (ce peut être, en particulier, une famille filtrante, à droite ou à gauche, auquel cas la 
limite est aussi bien enveloppe) converge simplement dans l'ouvert connexe $\Omega$, elle converge uniformément sur tout compact de $\Omega$ au sens de la structure uniforme de $\overline{\mathbf{R}}$; la limite est soit la constante $+\infty$, soit une minimale de $f$ (et, dans ce dernier cas, il est équivalent d'invoquer la structure uniforme additive de $\mathbf{R}$ ).

De tout cela découle, par le théorème d'Ascoli, des propriétés de compacité semblables au théorème de Montel.

1.b. Cette étude des familles de minimales est motivée par certains développements futurs de la théorie. Le présent exposé est en effet très loin d'épuiser la question. En premier lieu les applications mécaniques auxquelles nous ferons allusion plus loin conduisent à des problèmes aux limites plus généraux que le problème de Dirichlet. Il sera d'autre part nécessaire de définir et d'étudier les minimales d'une fonction $f$ non plus continue, mais seulement s.c.s., à valeurs dans $[-\infty,+\infty[$, définie dans $\Omega$ ou même plûtot s.c.s. dans le compact $\hat{\Omega}$ : à cet égard il est significatif de traiter le couple de données $\left(u^{*}, f\right)$ du " problème de Dirichlet » comme une seule fonction, s.c.s. dans $\hat{\Omega}$, qu'on peut d'ailleurs prolonger avec la valeur $-\infty$ hors de $\hat{\Omega}$, ce qui donne une fonction s.c.s. dans $\hat{\mathbf{R}}^{n}$ tout entier. L'une des applications mécaniques (théorie des membranes) pose d'ailleurs tout naturellement un problème plus général : au lieu d'une, on considère deux fonctions $f<g$, la première s.c.s., la seconde s.c.i. dans $\Omega$; on s'intéresse aux fonctions finies continues $u$ telles que $f \leqslant u \leqslant g$, admettant pour laplacien-distribution la somme de deux mesures: l'une $\geqslant 0$ ayant son support contenu dans l'ensemble $\{x: u(x)=g(x)\}$, l'autre $\leqslant 0$ ayant son support contenu dans l'ensemble $\{x: u(x)=f(x)\}$.

On a d'autre part laissé de côté un des aspects fondamentaux de la question : le point de vue "énergétique " dans lequel on invoque la norme de Dirichlet. On constatera, par exemple, que si les données sont assez régulières pour que la solution du " problème de Dirichlet" de tout à l'heure possède dans $\Omega$ un gradient de carré sommable, cette solution est caractérisée comme fournissant le minimum de l'intégrale de Dirichlet

$$
\int_{\Omega} \operatorname{grad}^{2} \varphi d x
$$


dans l'ensemble des fonctions $\varphi$ qui possèdent un gradient de carré sommable dans $\Omega$, majorent $f$, sont continues dans $\hat{\Omega}$ et admettent la donnée $u^{*}$ comme restriction à $\Omega^{*}$ (cf. Moreau [12]; cette approche conduit très naturellement au traitement simultané de paires de problèmes extrémaux en dualité dans un espace hilbertien : cf. Moreau [9] et [10]).

C'est sous cet aspect variationnel que les problèmes aux limites de ce genre ont été abordés, dans le style de la théorie moderne des équations aux dérivées partielles, par LionsStampacchia [8]. Ces auteurs montrent par là l'existence et l'unicité de la solution de problèmes affaiblis, solution définie comme élément d'un espace de Sobolev. Il reste ensuite à étudier la régularité d'une telle solution, ce à quoi s'emploient Brezis-Stampacchia [3], Lewy-Stampacchia [7]. Un type d'approche nettement différent est utilisé par Lewy [6] pour le cas particulier $n=2$ avec une fonction $f$ égale à $-\infty$ hors d'un segment de droite et de restriction continue à ce segment.

1.c. Indiquons enfin quels sont les problèmes de mécanique qui conduisent à l'étude de minimales d'une fonction $f$ donnée. Ils sont de deux sortes très différentes.

Le premier type de problème appartient à l'élastostatique et l'intérêt est limité à la dimension $n=2$. La fonction $u$ représente l'élongation transversale d'une membrane élastique homogène et isotrope supposée s'écarter infiniment peu d'un plan. Pour toute partie libre de la membrane la fonction $u$ est alors harmonique, tandis que, dans une partie soumise à une contrainte transversale, le laplacien $\Delta u$ est proportionnel à la pression subie par la membrane (comptée positivement s'il s'agit d'une pression proprement dite exercée dans le sens des $u$ décroissants). Le graphe de la fonction donnée $f$ représente la frontière d'un massif immuable qui repousse (sans frottement) la membrane en limitant inférieurement l'élongation $u$; il s'agit là d'une liaison unilatérale: la membrane peut se détacher de ce massif solide, mais elle ne peut le franchir. L'intégrale de Dirichlet $\int \operatorname{grad}^{2} u d x$ représente, à un coefficient positif près, l'énergie potentielle élastique de la membrane. 
De tels problèmes d'élastostatique avec liaisons unilatérales se présentent naturellement aussi en dimension 3, cela sous deux aspects principaux : il peut s'agir de milieux élastiques à trois dimensions confinés unilatéralement sur une partie de leur frontière (problème de Signorini, étudié principalement par Fichera [1], [5]) ou bien encore de liaisons unilatérales internes (milieux élastiques à blocage tels que le caoutchoucmousse, étudiés par Prager [14], [15]).

Un deuxième type de problème apparaît dans l'étude de la naissance de la casitation au sein d'un fluide parfait incompressible, telle qu'elle est abordée dans Moreau [11], [12]. La fonction numérique $u-f \geqslant 0$ représente alors, à un instant déterminé, la pression de ce fluide (ou plutôt l'excès de cette pression sur la pression de vapeur).

\section{Généralités.}

2.a. Notations. - On notera $\hat{\mathbf{R}}^{n}$ l'espace topologique compactifié de $\mathbf{R}^{n}$ par adjonction du point d'Alexandroff, noté $\infty$.

Par ouvert sauf précision contraire, on entendra toujours un ouvert de $\mathbf{R}^{n}$.

Pour toute partie $A$ de $\mathbf{R}^{n}$, on notera $\bar{A}$ l'adhérence et aA la frontière prises selon la topologie de $\mathbf{R}^{n}$. On notera $\hat{\mathbf{A}}$ l'adhérence et $\mathrm{A}^{*}$ la frontière prises selon la topologie de $\hat{\mathbf{R}}^{n}$. Autrement dit :

$$
\begin{aligned}
\hat{\mathrm{A}} & =\left\{\begin{array}{lll}
\overline{\mathrm{A}} & \text { si } & \text { A borné } \\
\overline{\mathrm{A}} \cup\{\infty\} & \text { si } & \mathrm{A} \text { non borné }
\end{array}\right. \\
\mathrm{A}^{*} & =\left\{\begin{array}{lll}
\partial \mathrm{A} & \text { si } & \mathrm{A} \text { borné } \\
\partial \mathrm{A} \cup\{\infty\} & \text { si } & \mathrm{A} \text { non borné. }
\end{array}\right.
\end{aligned}
$$

2.b. Définition. - Soit $\Omega$ un ouvert de $\mathbf{R}^{n}$ et soit $f$ une fonction, $\grave{a}$ valeurs dans $\mathbf{R}$, continue dans $\Omega$. On dira qu'une fonction $u$, à valeurs dans ] $-\infty,+\infty]$, est une majorante surharmonique minimale de $f$ dans $\Omega$ (ou, simplement, pour abréger, " une minimale de $f$ ) si cette fonction possède dans $\Omega$ les trois propriétés suivantes:

i) $u \geqslant f$

ii) $u$ est surharmonique (donc s.c.i.), 
iii) $u$ est "harmonique là où elle diffère de $f$ ", c'est-à-dire harmonique dans l'ouvert $\{x \in \Omega: u(x)>f(x)\}$.

Cet usage du mot minimale sera justifié à la section 5 .

Il s'agit visiblement là de propriétés locales : $u$ est minimale de $f$ dans $\Omega$ si et seulement si elle l'est dans chacun des ouverts d'une famille recouvrant $\Omega$.

En particulier, toute majorante harmonique de $f$ est une minimale de $f$.

Si $f$ est sousharmonique (ou, en particulier, harmonique) et si $u$ est une de ses minimales, $u-f$ est une fonction surharmonique $\geqslant 0$, donc partout nulle ou partout $>0$ dans chaque composante connexe de $\Omega$. Cela montre que, dans ce cas, toutes les minimales de $f$ sont harmoniques.

Si $f$ est surharmonique, elle constitue évidemment l'une de ses minimales.

Soit enfin $h$ une fonction harmonique dans $\Omega$; quelle que soit $f$, il est clair que $u$ est une minimale de $f$ si et seulement si $u+h$ est une minimale de $f+h$.

2.c. Proposition. - Toute minimale de $f$ est une fonction finie et continue dans $\Omega$.

En effet toute minimale $u$ de $f$ est, par définition, surharmonique; d'après le théorème de Riesz elle est, au moins localement, la somme d'une fonction harmonique (donc continue) et d'un potentiel de la mesure positive $-\Delta u / \varphi$ (avec $\varphi$ : coefficient numérique dépendant de la dimension $n$ ). Ce potentiel est continu dans le support de la mesure puisque la définition des minimales implique $u=f$ dans ce support; il est donc continue partout, d'après le théorème d'EvansVasilesco (cf. Brelot, [2], chap. Iv, §5), ce qui prouve la continuité de $u$.

Remarque. - Si l'on avait posé à priori la continuité de $u$, la condition ii) de la définition $2 . b$ aurait pu encore s'écrire

$$
\Delta u \leqslant 0,
$$

notation signifiant que le laplacien-distribution de $u$ est une distribution négative, c'est-à-dire une mesure négative. Et la condition iii) de cette même définition s'écrivait alors

$$
(u-f) \Delta u=0 \text {, }
$$

c'est-à-dire que le produit de la mesure $\Delta u$ par la fonction 
continue $u-f$ est la mesure nulle dans $\Omega$. Il faut et suffit pour cela que $u-f$ (par hypothèse $\geqslant 0$ ) soit nulle dans le support (relativement à $\Omega$ ) de la mesure négative $\Delta u$.

\section{2.d. Cas : $n=1$.}

En dimension 1, fonction surharmonique signifie fonction concave; fonction harmonique signifie fonction affine. Une minimale de $f$ est ainsi, dans ce cas, une majorante concave de cette fonction possédant la propriété d'être affine là où elle diffère de $f$.

Considérons l'hypographe de $f$, c'est-à-dire l'ensemble

$$
\mathrm{F}=\{x, r) \in \Omega \times \mathbf{R}: f(x) \geqslant r\} .
$$

Le graphe d'une minimale $u$ de $f$ est facile à dessiner comme étant une réunion d'arcs du graphe de $f$ et de segments de droites d'appui de l'ensemble F (fig. ci-dessous).

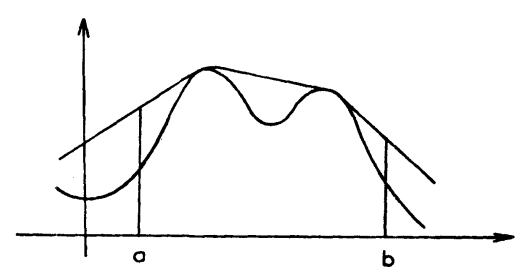

La théorie élémentaire de la convexité fournit, dans ce cas $n=1$, la démonstration facile de faits dont on retrouvera les analogues en dimension quelconque. Soit, par exemple, $[a, b]$ un intervalle fermé contenu dans $\Omega$; la restriction à $[a, b]$ de la fonction $u$, minimale quelconque de $f$, constitue la plus petite fonction concave majorant $f$ sur cet intervalle et prenant aux extrémités les valeurs $u(a)$ et $u(b)$. Si l'ensemble $\mathrm{F}$ est réalisé matériellement, on peut concevoir le graphe de $u$ comme un fil, tendu entre les deux points $(a, u(a))$ et $(b, u(b))$, fil contournant par en dessus l'ensemble $\mathrm{F}$. Une interprétation mécanique analogue peut être donnée pour $n=2$ (membranes, dans le cadre d'une théorie linéarisée); ce n'est là qu'une des motivations mécaniques de la présente théorie.

2.e. Donnons maintenant, pour $n$ quelconque, un exemple "calculable " de minimale. Cet exemple est très utile pour tester des techniques numériques (cf. Durand [4]). 
Soit $h$ une fonction harmonique dans $\Omega$; soit $\varphi$ une fonction réelle continue d'une variable réelle, définie au moins sur l'ensemble des valeurs de $h$ et soit $\psi$ une minimale de $\varphi$, (construite comme on l'explique au paragraphe précédent).

La fonction $u=\psi \circ h$ est une minimale de $f=\varphi \circ h$.

En effet $\psi$ étant concave et $h$ harmonique, il est classique que $\psi \circ h$ est surharmonique et elle majore $\varphi \circ h$ puisque $\psi$ majore $\varphi$. Soit $x_{0} \in \Omega$ tel que $\psi\left(h\left(x_{0}\right)\right)>\varphi\left(h\left(x_{0}\right)\right)$; puisque $\varphi$ et $\psi$ sont continues, il existe un intervalle $] h\left(x_{0}\right)-\varepsilon$, $h\left(x_{0}\right)+\varepsilon[$ sur lequel $\psi$ majore strictement $\varphi$; la restriction de $\psi$ à l'intervalle en question est affine. En outre, par la continuité de $h$, il existe un voisinage ouvert $\omega$ de $x_{0}$ sur lequel $h$ prend des valeurs contenues dans l'intervalle cidessus. La fonction $\psi \circ h$ est harmonique dans $\omega$ comme fonction affine d'une fonction harmonique; elle est donc bien harmonique dans l'ouvert où elle diffère de $\varphi \circ h$.

\section{Comparaisons de minimales.}

3.a. Proposition. - Si $u$ et $\rho$ sont deux minimales de $f$, la fonction $|u-\varphi|$ est sousharmonique.

En effet, dans l'ouvert

$$
\{x \in \Omega: u(x)-v(x)>0\},
$$

on a nécessairement $u>f$, donc $u$ harmonique; par conséquent $|u-\vartheta|=u-\vartheta$ est sousharmonique dans cet ouvert. De la même manière, dans l'ouvert

$$
\{x \in \Omega: \varphi(x)-u(x)>0\},
$$

$|u-\varphi|=\varphi-u$ est sousharmonique. Dans le reste de $\Omega$, on a $|u-\rho|=0$, donc en tout point de $\Omega$ la fonction continue $|u-\vartheta|$ est inférieure ou égale à sa moyenne dans une boule suffisamment petite centrée en ce point.

3.b. Proposition. - Si u est une minimale de $f$ et si $g$ est une fonction continue telle que $f \leqslant g \leqslant u$ dans $\Omega$, alors $u$ est une minimale de $g$.

En effet $u$ est bien une majorante surharmonique de $g$ et l'ouvert

$$
\{x \in \Omega: u(x)>g(x)\}
$$


est contenu dans l'ouvert

$$
\{x \in \Omega: u(x)>f(x)\},
$$

où $u$ est harmonique par hypothèse.

En rapprochant ce résultat de la Proposition 3.a on obtient :

Corollaire. - Soient $u$ et $v$ deux minimales de f. Si $u \geqslant \varphi$ dans $\Omega$, la fonction $u-\varphi$ est sousharmonique et $u$ est une minimale de $\varphi$.

3.c. Proposition. - Toute minimale u d'une minimale $\bullet$ de $f$ est une minimale de $f$.

En effet $\varphi$ est elle-même une de ses minimales; le corollaire ci-dessus montre donc que $u-\varphi$ est sousharmonique. On écrit alors (cf. Remarque 2.c) :

$$
\begin{aligned}
(u-f) \Delta u & =(u-\varphi) \Delta u+(\vartheta-f) \Delta \vartheta+(\vartheta-f) \Delta(u-\vartheta) \\
& =(\vartheta-f) \Delta(u-\vartheta) .
\end{aligned}
$$

Le premier membre est une mesure $\leqslant 0$ et le dernier membre une mesure $\geqslant 0$ : cela montre la nullité de $(u-f) \Delta u$.

\section{Minimalisation.}

4.a. Harmonisation conditionnelle sur une boule.

Supposons qu'il existe une fonction $\varphi$, surharmonique dans $\Omega$, majorant $f$. Soit $B$ une boule ouverte, d'adhérence $\bar{B}$ contenue dans $\Omega$. Notons $\varphi_{B}$ la fonction égale à $\varphi$ dans $\Omega \backslash B$ et égale dans $B$ à l'intégrale de Poisson $I_{v}^{\mathrm{B}}$; on sait (cf. Brelot [2], chap. II, $\S 1$ ) que cette fonction est encore surharmonique et qu'elle minore $\varphi$ dans $\Omega$; elle est d'ailleurs continue si $v$ est continue. Il se peut qu'elle ne majore plus $f$; convenons alors de la notation $\hat{Q}_{\mathrm{B} / \mathcal{S}}$ pour désigner :

- la fonction $\varphi_{\mathrm{B}}$ si celle-ci majore $f$ partout dans $\Omega$;

- la fonction $\varphi$ dans le cas contraire.

La substitution de $\varphi_{\mathrm{B} / f}$ à $\rho$ est une opération qu'on peut intituler harmonisation de $\diamond$ dans $\mathrm{B}$, conditionnelle relativement à $f$. Partant de $\varphi$, majorante surharmonique de $f$, cette opération conduit encore à une majorante surharmonique de $f$, minorant $\varphi$. 
Proposition. - Si u est minimale de f, alors $u_{\mathrm{B} / f}=u$.

Ce n'est là qu'un cas particulier de propriétés qu'on établira à la section 5; pour l'instant on peut raisonner comme suit : Puisque $u$ est continue, il en est de même de $u_{\mathrm{B} / f}$; la Proposition $3 . b$ montre que $u$ est minimale de $u_{\mathrm{B} / f}$ et cette dernière est minimale d'elle-même, puisque surharmonique. Alors la Proposition 3.a montre que la différence $u-u_{\mathrm{B} / f} \geqslant 0$ est sousharmonique. Essentiellement nulle dans $\Omega \backslash B$, cette différence est aussi nulle dans $B$ puisque nulle sur la sphère oB.

4.b. Soit $\left(\beta_{n}\right)$ une suite de boules ouvertes, d'adhérences $\left(\bar{\beta}_{n}\right)$ contenues dans $\Omega$, constituant une base de la topologie de $\Omega$. On construit une suite double $\left(\beta_{i j}^{\prime}\right)$ en posant

$$
\beta_{i j}^{\prime}=\beta_{i} \quad(i \in \mathbf{N}, j \in \mathbf{N}) \text {. }
$$

Notons $\left(B_{n}\right)$ la suite simple obtenue en réindexant cette suite double par le procédé classique, c'est-à-dire que

représente la suite

$$
B_{1}, B_{2}, B_{3}, \ldots
$$

$$
\beta_{1}, \beta_{2}, \beta_{1}, \beta_{2}, \beta_{3}, \beta_{1}, \beta_{2}, \beta_{3}, \beta_{4}, \ldots
$$

Bref, chaque élément de la suite $\left(\beta_{n}\right)$ figure une infinité de fois dans la suite $\left(B_{n}\right)$.

Soit $q$ une majorante surharmonique de $f$ dans $\Omega$ (s'il en existe). Posons $\varphi_{1}=\varphi_{\mathrm{B}_{1} / f}$ et construisons par récurrence une suite de majorantes surharmoniques de $f$ en appelant $\varphi_{n}$ la fonction déduite de $\varphi_{n-1}$ par harmonisation dans $B_{n}$, conditionnelle relativement à $f$; cette suite est décroissante. La limite $\varphi_{\infty}$ (ou enveloppe inférieure) des $\varphi_{n}$ est une fonction surmédiane (cf. Brelot [2], chap. $2, \S 9$ ) qui majore $f$. Notons $\bar{\varphi}$ la régularisée semi-continue inférieurement de cette limite: c'est une fonction surharmonique et elle majore encore $f$ puisque cette dernière est continue. On va montrer:

\footnotetext{
Proposition. - La fonction $\bar{\varphi}$ est une minimale de $f$ dans $\Omega$.

Il reste seulement à prouver que $\bar{\varphi}$ est harmonique dans l'ouvert

$$
\omega=\{x \in \Omega: \bar{\varphi}(x)-f(x)>0\} .
$$
}


Soit $x_{0} \in \omega$; les boules $\beta_{n}$ constituent une base de la topologie de $\Omega$; soit $\beta$ l'une d'entre elles telle que

$$
x_{0} \in \beta \subset \bar{\beta} \subset \omega .
$$

Il résulte de la semi-continuité inférieure de $\bar{\rho}$ et de la continuité de $f$ qu'on peut de plus choisir $\beta$ assez petite pour que

$$
\inf \bar{\varphi}(\bar{\beta}) \geqslant \sup f(\bar{\beta}) \text {. }
$$

Ayant ainsi choisi $\beta$, extrayons de la suite $\varphi_{n}$ une sous-suite en ne retenant que les valeurs de $n$ telles que

$$
\mathrm{B}_{n}=\beta \text {. }
$$

Pour toutes ces valeurs de $n$, l'inégalité (3.1) montre que

$$
\inf \varphi_{n-1}(\overline{\mathrm{B}}) \geqslant \sup f\left(\overline{\mathrm{B}}_{n}\right) \text {; }
$$

donc l'intégrale de Poisson de $\varphi_{n-1}$ sur la sphère $\partial \mathrm{B}_{n}$ est une fonction définie dans la boule $\mathrm{B}_{n}$, majorant $f$ dans cette boule, ce qui signifie que l'harmonisation conditionnelle est, dans ce cas, une harmonisation effective : $\varphi_{n}$ est harmonique dans $\mathrm{B}_{n}$. On a donc extrait de la suite convergente $\varphi_{n}$ une sous-suite décroissante de fonctions harmoniques dans $\beta$; la limite $\varphi_{\infty}$ est ainsi harmonique dans $\beta$, donc continue, donc égale dans cette boule à sa régularisée s.c.i., c'est-à-dire $\bar{\varphi}$. On prouve ainsi que cette dernière est harmonique dans $\omega$.

4.c. La propriété que voici montre que, $\varphi$ étant une majorante surharmonique quelconque de $f$, la minimale $\bar{\varphi}$ qu'on lui associe par la construction précédente est indépendante $d u$ choix de la suite de boules $\left(\beta_{n}\right)$.

Proposition. - La fonction $\bar{\varphi}$ est (pour l'ordre naturel des fonctions numériques dans $\Omega$ ) la plus grande minimale de $f$ minorant $\bullet$. On l'appellera la minimalisée de $\diamond$ relativement $\grave{a} f$.

En effet, soit $u$ une minimale de $f$ minorant $\varphi$; l'isotonie de l'intégrale de Poisson montre que

$$
u_{\mathrm{B} / f} \leqslant \varphi_{\mathrm{B} / f}
$$

Comme $u_{\mathrm{B} / f}=u$ (Proposition 4.a), on voit, de proche en proche, que $u$ minore toutes les $\varphi_{n}$, donc leur limite $\varphi_{\infty}$ 
et donc aussi, puisque $u$ est continue, la fonction $\bar{\rho}$ qui est la régularisée s.c.i. de cette limite.

4.d. Supposons que $f$ possède des majorantes surharmoniques. L'enveloppe inférieure de ces majorantes est une fonction surmédiane majorant $f$. La régularisée s.c.i. de cette enveloppe majore encore $f$ puisque cette dernière est continue. Cette régularisée est de la sorte, la plus petite majorante surharmonique de $f$, donc nécessairement égale à sa minimalisée. C'est ainsi une minimale de $f$; en invoquant de plus les Propositions 3.a, 3.b, 3.c, on obtient :

Proposition. - Si $f$ (continue dans $\Omega$ ) possède des majorantes surharmoniques, il en existe une plus petite que toute autre, notée $\hat{f}$; c'est une minimale de $f$. Une fonction $u$ est minimale de $f$ si et seulement si elle est minimale de $\hat{f}$ et, en ce cas, la différence $u-\hat{f}$ est une fonction sousharmonique $\geqslant 0$ continue.

4.e. Proposition. - Dans l'ensemble $\mathrm{M}(f)$ des minimales de $f$, ordonné par l'ordre naturel des fonctions numériques sur $\Omega$, toute partie non side possède un plus grand minorant, toute partie majorée possède un plus petit majorant.

En effet, si A est une partie non vide de $\mathrm{M}(f)$, l'enveloppe inférieure des fonctions de A est une fonction surmédiane majorant $f$. Soit $\diamond$ la régularisée s.c.i. de cette enveloppe : c'est une majorante surharmonique de $f$. Une fonction $u \in \mathrm{M}(f)$ minore $\mathrm{A}$ si et seulement si elle minore $\varphi$. D'après la Proposition 4.c, la minimalisée de $\rho$ est le plus grand élément de $\mathrm{M}(f)$ possédant cette propriété.

Supposons en outre que A possède un majorant dans $\mathrm{M}(f)$; l'ensemble $\mathrm{A}^{\prime}$ des éléments de $\mathbf{M}(f)$ majorant $\mathrm{A}$ est alors non vide et possède d'après ce qui précède un plus grand minorant; ce plus grand minorant est, en particulier, plus grand que tous les éléments de $\mathrm{A}$ : il appartient donc à $\mathrm{A}^{\prime}$ et en est le plus petit élément.

Remarque. - Pour que A soit majoré dans $\mathrm{M}(f)$, il suffit, d'après la proposition 4.c, qu'il existe une fonction surharmonique majorant toutes les fonctions de $\mathrm{A}$. 


\section{Un ordre sur l'ensemble des majorantes surharmoniques de $f$.}

5.a. Notation. - Soient $u$ et $\varphi$ deux fonctions numériques définies dans l'ouvert $\Omega$. On exprimera par l'écriture $\varphi \nmid u$ la propriété suivante : il existe un ouvert borné $\omega$ tel que $\bar{\omega} \subset \Omega$ et tel qu'on ait

$$
\begin{array}{lll}
\varphi \leqslant u & \text { dans } & \omega \\
\varphi=u & \text { dans } & \Omega \backslash \omega .
\end{array}
$$

On vérifie immédiatement que la relation $\prec$ constitue un ordre dans l'ensemble $\overline{\mathbf{R}}^{\Omega}$ des applications de $\Omega$ dans $\overline{\mathbf{R}}$.

Proposition. - Soit F l'ensemble des fonctions surharmoniques dans $\Omega$, majorant la fonction finie continue $f$ (au sens de la relation d'ordre classique des fonctions numériques). Dans F, ordonné par $\prec$, une fonction $u$ constitue un élément minimal si et seulement si $u$ est une minimale de $f$ au sens de la Définition 2.b.

Démonstration. $1^{0}$ Supposons que $u$ soit élément minimal dans $(\mathrm{F}, \prec)$. Les notations posées en $4 . a$ impliquent $u_{\mathrm{B} / f} \in \mathrm{F}$ et (en prenant $\omega=\mathrm{B}$ ) que

$$
u_{\mathrm{B} / f} \prec u,
$$

donc $u_{\mathrm{B} / f}=u$. Il en résulte que toutes les $u_{n}$ qu'on associerait à $u$ par la construction du paragraphe $4 . b$ lui sont égales, de même que leur limite $u_{\infty}$. Cette limite est donc s.c.i., donc égale à la fonction, notée $\bar{u}$, " minimalisée » de $u$. Cela prouve que $u$ est une minimale de $f$.

$2^{\circ}$ Appelons maintenant $u$ une minimale de $f$. Soit $\varphi \in \mathrm{F}$ tel que $\diamond \prec u$ et soit $\omega$ l'ouvert impliqué par cette écriture. Il faut montrer que $\rho=u$ dans $\Omega$, ce qui est déjà assuré dans $\Omega \backslash \omega$. Dans l'ouvert

$$
\omega^{\prime}=\{x \in \omega: u(x)>f(x)\},
$$

$u$ est harmonique, donc $\varphi-u$ surharmonique: le principe du minimum montrera que cette différence est $\geqslant 0$ dans $\omega^{\prime}$ 
si on prouve que pour tout $x_{0}$, point frontière de $\omega^{\prime}$, on a $\lim$.inf $(\varphi-u) \geqslant 0$ selon le filtre des traces sur $\omega^{\prime}$ des voisinages de $x_{0}$. Comme $\varphi-u$ est s.c.i. dans $\Omega$ il suffit pour cela de prouver que, pour tout $x_{0} \in \partial \omega^{\prime}$ on a

$$
\varphi\left(x_{0}\right)-u\left(x_{0}\right) \geqslant 0 .
$$

De fait on distingue les deux cas suivants :

- si $x_{0} \notin \omega$ on a $v\left(x_{0}\right)-u\left(x_{0}\right)=0$ d'après (5.2);

- si $x_{0} \in \omega$ on a $u\left(x_{0}\right)=f\left(x_{0}\right)$ d'après la définition de $\omega^{\prime}$, donc $\varphi\left(x_{0}\right) \geqslant u\left(x_{0}\right)$.

Enfin, dans $\omega \backslash \omega^{\prime}$ on a $u=f$ donc $\rho \geqslant u$.

Bref $\varphi \geqslant u$ partout dans $\Omega$, ce qui, rapproché, de (5.1) et (5.2) montre que $\varphi=u$; c.q.f.d.

5.b. Remarque. - Soit $\mathscr{B}$ une famille d'ouverts, d'adhérences contenues dans $\Omega$ et constituant une base de la topologie de $\Omega$. On pourrait exprimer par l'écriture $\varphi \prec_{\mathscr{B}} u$ la propriété suivante : il existe $\omega \in \mathscr{B}$ tel que l'on ait (5.1) et (5.2). C'est visiblement là une relation d'ordre moins fine que $\prec$; par conséquent la proposition précédente implique que toute minimale de $f$ est aussi élément minimal dans l'ensemble ordonné $\left(\mathrm{F}, \prec_{\mathfrak{B}}\right)$. Mais réciproquement, un tel élément minimal est nécessairement une minimale de $f$ : il suffit pour le voir de reprendre le $1^{0}$ de la démonstration précédente en remarquant que la construction $4 . b$ peut être menée à bien en retenant seulement dans la suite $\left(\beta_{n}\right)$ les boules $\beta$ possédant la propriété «il existe un ensemble de $\mathscr{B}$ contenant $\beta$ »; les boules ainsi retenues forment bien encore une base de la topologie de $\Omega$.

\section{Problème de Dirichlet pour les minimales.}

6.a. Par problème de Dirichlet pour les minimales de la fonction continue $f$ dans $\Omega$, nous entendrons (on rappelle que $\hat{\Omega}$ et $\Omega^{*}$ désignent respectivement l'adhérence et la frontière de $\Omega$ par rapport à l'espace compactifié $\left.\hat{\mathbf{R}}^{n}\right)$ :

Proвlème. - On donne dans $\Omega^{*}$ une fonction numérique finie $u^{*}$. Trouver $u$, fonction numérique finie et continue dans 
MAJORANTES SURHARMONIQUES MINIMALES D'UNE FONCTION 143

$\hat{\Omega}$, minimale de $f$ dans $\Omega$ et admettant $u^{*}$ comme restriction $\grave{a} \Omega^{*}$.

Les données $u^{*}$ et $f$ doivent vérifier les conditions de possibilité évidentes :

$10 u^{*}$ continue dans $\Omega^{*}$,

$2^{\circ}$ pour tout $a \in \Omega^{*}$

$$
\lim _{x \rightarrow a} \sup f(x) \leqslant u^{*}(a),
$$

qui seront toujours supposées remplies par la suite. On notera $f^{*}(a)$ le premier membre de (6.1).

Remarque 1. - Il serait équivalent d'imposer à $u$, définie seulement dans $\Omega$ et minimale de $f$, la condition :

$$
\lim _{x \rightarrow a} u(x)=u^{*}(a)
$$

pour tout $a \in \Omega^{*}$ (l'existence d'un tel $u$ exige toujours que $u^{*}$ soit continue dans $\Omega^{*}$ puisqu'il s'agit de fonctions à valeurs dans $\mathbf{R}$, espace régulier).

Remarque 2. - S'il existe une fonction $h$ harmonique dans $\Omega$, continue dans $\hat{\Omega}$ et admettant $u^{*}$ comme restriction à $\Omega^{*}$ (solution du problème de Dirichlet classique), la fonction $u$ cherchée peut être caractérisée par le fait que $u-h$ est minimale de $f-h$ (cf. remarque finale du $\S 2 . a$ ), continue dans $\hat{\Omega}$ et nulle dans $\Omega^{\star}$.

6.b. La propriété suivante montre que le problème en question possède au plus une solution.

Proposition. - Si le problème de Dirichlet 6.a possède une solution $u$, cette fonction est le plus petit élément dans l'ensemble des fonctions $\varphi$, s.c.i. dans $\hat{\Omega}$, surharmoniques et majorant $f$ dans $\Omega$, telles enfin que $\varphi \geqslant u^{*}$ dans $\Omega^{*}$.

La démonstration est très semblable à celle de la Proposition 5. $a, 2^{\circ}$ :

Soit $\checkmark$ possédant les propriétés énoncées; il faut montrer que $\varphi \geqslant u$ dans $\Omega$. En effet, dans l'ouvert

$$
\omega=\{x \in \Omega: u(x)>f(x)\},
$$

$u$ est harmonique, donc $v-u$ est surharmonique. Cette 
différence étant s.c.i. dans $\hat{\Omega}$, le principe du minimum prouvera qu'elle est $\geqslant 0$ dans $\omega$ si on montre que pour tout $x_{0} \in \omega^{*}$, on a

$$
\varphi\left(x_{0}\right)-u\left(x_{0}\right) \geqslant 0 .
$$

Or, si $x_{0} \in \Omega^{*}$ cela résulte de l'hypothèse; si $x_{0} \in \Omega$, on a $u\left(x_{0}\right)=f\left(x_{0}\right)$ d'après la définition de $\omega$, donc encore (6.2) puisque $\varphi$ majore $f$. Pour la même raison on a $\varphi \geqslant u$ dans $\Omega \backslash \omega$.

On tire de la proposition précédente l'isotonie de la solution $u$ à l'égard des données $u^{*}$ et $f$ :

Corollaire 1. - Si $u_{1}^{*}$ et $u_{2}^{*}$ sont deux fonctions finies et continues dans $\Omega^{*}$ telles que $u_{1}^{*} \geqslant u_{2}^{*}$, les solutions correspondantes $u_{1}$ et $u_{2} d u$ problème $6 . a$, si elles existent, satisfont la même inégalité partout dans $\hat{\Omega}$.

En effet $u_{1}$ est une fonction continue dans $\hat{\Omega}$, surharmonique et majorant $f$ dans $\Omega$, telle enfin que $u_{1} \geqslant u_{2}^{*}$ dans $\Omega^{*}$.

Corollaire 2. - Si, pour une même donnée $u^{*}$ dans $\Omega^{*}$, les solutions $u_{1}$ et $u_{2}$ du Problème 6.a correspondant à deux fonctions continues $f_{1}$ et $f_{2}$ existent et si $f_{1} \geqslant f_{2}$ dans $\Omega$, on $a \quad u_{1} \geqslant u_{2}$ dans $\hat{\Omega}$.

Car $u_{1}$ est une fonction continue dans $\hat{\Omega}$, surharmonique et majorant $f_{2}$ dans $\Omega$, égale à $u_{2}$ dans $\Omega^{*}$.

6.c. Quant à l'existence de la solution, on a :

Proposition. - Si l'ouvert $\Omega$ est régulier pour le problème de Dirichlet classique (celui des fonctions harmoniques), le Problème 6.a possède une solution quelle que soit la donnée $u^{*}$, fonction finie continue dans $\Omega^{*}$ vérifiant la condition (6.1).

On décompose la démonstration comme suit:

Lemme 1. - $\Omega$ étant supposé régulier pour le problème de Dirichlet classique, si en tout point de $\Omega^{*}$ la fonction finie et continue $u^{*}$ majore strictement la fonction

$$
y \longmapsto f^{*}(y)=\lim _{x \rightarrow y} \sup f(x),
$$


$u^{*}$ possède un prolongement $\bullet$ continu dans $\hat{\Omega}$ surharmonique et majorant $f$ dans $\Omega$.

En effet, soit $h$ la solution du problème de Dirichlet classique, fonction harmonique dans $\Omega$, continue dans $\hat{\Omega}$, égale à $u^{*}$ dans $\Omega^{*}$. Si $h$ majore $f$ partout, cette fonction constitue le prolongement cherché (et c'est d'ailleurs dans ce cas la solution du Problème 6.a). Sinon, désignons encore par $f$ la fonction définie dans $\hat{\Omega}$ en prolongeant $f$ par $f^{*}$ : c'est une fonction s.c.s. dans $\hat{\Omega}$ de sorte que l'ensemble non vide

$$
\mathrm{K}=\{x \in \hat{\Omega}: h(x)-f(x) \leqslant 0\}
$$

est fermé dans $\hat{\Omega}$, donc compact. Cet ensemble est contenu dans $\Omega$ d'après l'hypothèse.

Soit $s$ une fonction surharmonique continue dans $\hat{\Omega}$, nulle sur $\Omega^{*}$, strictement positive dans $\Omega$ (c'est, par exemple, le potentiel de Green dans $\hat{\Omega}$ d'une densité $\geqslant 0$, continue à support compact dans $\Omega$, de restriction non nulle à chaque composante connexe de $\Omega$ : ce potentiel est bien continu dans $\hat{\Omega}$ et nul à la frontière parce qu'on a supposé $\Omega$ régulier pour le problème de Dirichlet). Soit $\lambda$ une constante $>0$; en tout point de $\mathrm{K}$ on a

$$
h+\lambda s \geqslant \min _{\mathbf{K}} h+\lambda \min _{\mathbf{K}} s .
$$

Comme $\min _{\mathbf{K}} s>0$, on pourra choisir $\lambda$ assez grand pour que le deuxième membre soit supérieur à $\max _{\mathbf{K}} f$. Dès lors $h+\lambda s$ majore $f$ partout dans $\Omega$ et constitue le prolongement cherché.

Lem ме 2. - Dans les conditions du Lemme 1, la minimalisée $\bar{\sigma}$ (cf. 4.c) de $\varphi$ se prolonge continûment en une fonction égale à $u^{*}$ dans $\Omega^{*}$ et fournit donc la solution du Problème 6.a.

C'est bien en effet une minimale de $f$; elle minore $v$ et, d'autre part, elle majore $h$ car $\varphi$ majore $h$ de même que toutes les fonctions $\varphi_{n}$ et $\varphi_{\infty}$ introduites au $\S 4 . b$ pour la construction de $\bar{\varphi}$. Comme $\varphi$ et $h$ sont continues dans $\hat{\Omega}$, de même limite $u^{*}$ à la frontière $\Omega^{*}$, la fonction $\bar{v}$ admet cette même limite à la frontière. 
Pour achever d'établir la Proposition 6.c, on note que si la fonction finie $u^{*}$ ne majore pas strictement la fonction $f^{*}$ invoquée au Lemme 1, il en sera tout au moins ainsi pour

$$
u_{n}^{*}=u^{*}+\frac{1}{n}
$$

qui décroît et tend uniformément vers $u^{*}$ lorsque l'entier $n$ tend vers $+\infty$; on s'appuiera alors sur:

Lemme 3. - Soit $u_{n}$ la solution du Problème 6.a pour la donnée frontière $u_{n}^{*}$ définie en (6.3). La suite $u_{n}$ converge uniformément dans $\hat{\Omega}$ vers une fonction $u$ qui est solution $d u$ Problème 6.a pour la donnée $u^{*}$.

En effet, si $i \geqslant j$ le Corollaire $1 \mathrm{du} \S 6 . b$. montre que $u_{i} \leqslant u_{j}$ partout dans $\hat{\Omega}:$ la suite $u_{n}$ est décroissante. La proposition $3 . b$ montre alors que la différence $u_{j}-u_{i} \geqslant 0$ est sous-harmonique dans $\Omega$ donc partout inférieure à la valeur $1 / j-1 / i$ qu'elle prend à la frontière. Ainsi la suite est uniformément de Cauchy, donc uniformément convergente dans $\hat{\Omega}$; cela montre la continuité de la limite $u$. Cette limite est donc surharmonique et majore $f$, comme chacun des $u_{n}$. Sur l'ensemble ouvert

$$
\omega=\{x \in \Omega: u(x)-f(x)>0\}
$$

chacune des $u_{n}$ majore strictement $f$; comme cette fonction est une minimale de $f$, elle est harmonique dans $\omega$; il en est de même de $u$, limite d'une suite décroissante de fonctions harmoniques. Cela prouve que $u$ est minimale de $f$ et achève la démonstration de la Proposition 6.c.

6.d. Les résultats qui vont suivre précisent la dépendance de la solution $u$ d'un problème de Dirichlet à l'égard de sa donnée $u^{*}$.

Proposition. - Soit $\Omega$ un ouvert régulier pour le problème de Dirichlet et soient $u$ et $\rho$ deux minimales de $f$ dans $\Omega$ admettant des fonctions limites $u^{*}$ et $\varphi^{*}$ (nécessairement continues) sur la frontière $\Omega^{*}$. La solution $i$ du Problème 6.a pour la donnée

$$
i^{*}=\inf \left(u^{*}, \iota^{*}\right)
$$


est la plus grande minimale de $f$ minorant $u$ et $\varphi$ dans $\Omega$. La solution $s$ pour la donnée frontière

$$
s^{*}=\sup \left(u^{*}, \rho^{*}\right)
$$

est la plus petite minimale de $f$ majorant $u$ et $\diamond$ dans $\Omega$.

En effet, d'après 6.b, Corollaire 1, la minimale $i$ minore $u$ et $\nu$; elle minore donc la plus grande minimale minorant $u$ et $\varphi$, c'est-à-dire la minimalisée de la fonction surharmonique inf $(u, \varphi)$ (cf. Proposition 4.c); on voit comme au paragraphe précédent (démonstration du Lemme 2) que cette minimalisée a pour limite $i^{*}$ sur $\Omega^{*}$; elle coïncide donc avec $i$.

Soit, d'autre part, $\leftrightarrow$ une minimale de $f$ dans $\Omega$ majorant $u$ et $\varphi$. En associant à chaque point $x_{0}$ de $\Omega^{*}$ la limite inférieure de $w$ selon le filtre des traces sur $\Omega$ des voisinages de $x_{0}$, on prolonge cette fonction en une fonction notée encore $\{$ qui est la plus grande fonction s.c.i. dans $\hat{\Omega}$, minorant $\leftrightarrow$ dans $\Omega$. Elle majore donc $u$ et $\varphi$ dans $\hat{\Omega}$, c'est-à-dire que sa restriction à $\Omega^{*}$ majore $s^{*}$. D'après la Proposition 6.b, la fonction $w$ majore $s$.

6.e. La fonction $f$ étant toujours supposée fixée, on va considérer la donnée à la frontière $u^{*}$ comme un élément de l'espace de Banach $\mathfrak{C}\left(\Omega^{*}\right)$ : ensemble des fonctions numériques finies et continues sur le compact $\Omega^{*}$, ensemble muni de la norme de la convergence uniforme.

L'ensemble $\mathrm{C}_{f}$ des $u^{*}$ qui vérifient la condition de possibilité (6.1) est convexe fermé dans $\mathfrak{C}\left(\Omega^{*}\right)$; en effet, pour chaque $a \in \Omega^{*}$ l'application $u^{*} \longmapsto u^{*}(a)$ est une forme linéaire continue dans $\mathfrak{C}\left(\Omega^{*}\right)$.

Proposition. - On suppose $\Omega$ régulier pour le problème de Dirichlet classique et on note $u$ la solution du Problème 6.a correspondant à une donnée à la frontière $u^{*}$. Alors pour chaque $x_{0} \in \Omega$, l'application $u^{*} \longmapsto u\left(x_{0}\right)$ de $\mathrm{C}_{f}$ dans $\mathbf{R}$ est convexe et continue.

La continuité, pour la topologie de la norme $\|\cdot\|$ de $\mathfrak{C}\left(\Omega^{*}\right)$, résulte de la Proposition 3.a et du principe du maximum pour les fonctions sousharmoniques lequel implique, si $u_{1}$ et $u_{2}$ sont deux minimales de $f$ admettant respectivement $u_{1}^{*}$ 
et $u_{2}^{*}$ comme fonctions limite à la frontière

$$
\left|u_{1}\left(x_{0}\right)-u_{2}\left(x_{0}\right)\right| \leqslant\left\|u_{1}^{*}-u_{2}^{*}\right\| .
$$

La convexité résulte de ce que, si $\alpha_{1}$ et $\alpha_{2}$ sont des constantes dans ]0,1[, avec $\alpha_{1}+\alpha_{2}=1$, la fonction $\alpha_{1} u_{1}+\alpha_{2} u_{2}$ est une majorante surharmonique de $f$, continue dans $\hat{\Omega}$ et admettant $\alpha_{1} u_{1}^{*}+\alpha_{2} u^{*}$ comme fonction limite à la frontière. Alors la Proposition 6.b montre que, si $u_{3}$ est la solution du Problème 6. $a$ pour cette donnée à la frontière on a $u_{3} \leqslant \alpha_{1} u_{1}+\alpha_{2} u_{2}$ partout dans $\Omega$.

Variante 1. - On peut aussi considérer $u$ comme un élément de l'espace $\mathfrak{C}(\hat{\Omega})$, muni de la norme de la convergence uniforme: l'inégalité (6.4) montre que l'application $u^{*} \longmapsto u$ est continue de $\mathrm{C}_{f} \subset \mathcal{C}\left(\Omega^{*}\right)$ dans $\mathfrak{C}(\hat{\Omega})$; en outre, $\mathfrak{e}(\hat{\Omega})$ étant muni de l'ordre naturel, cette application est convexe.

Variante 2. - On peut convenir que si la donnée $u^{*}$ ne vérifie pas (6.1), la solution $u$ correspondante est la constante $+\infty$. Cette convention parait naturelle si on envisage que, dans le cas considéré jusqu'à présent, $u$ se trouve ètre l'inf de la famille des majorantes surharmoniques continues de $f$ ayant $u^{*}$ pour restriction à $\Omega^{*}$; si $u^{*} \notin \mathrm{C}_{f}$ cette famille est vide de sorte que son inf est bien $+\infty$. Par cette convention l'application $u^{*} \longmapsto u\left(x_{0}\right)$ se trouve définie dans $\mathfrak{C}\left(\Omega^{*}\right)$ tout entier: c'est alors une fonction à valeurs dans ]$-\infty,+\infty]$, convexe et s.c.i. (puisque $\mathrm{C}_{f}$ est convexe fermé), telle qu'on en considère dans la théorie générale des fonctionnelles convexes (cf. Moreau [13]). Cette fonctionnelle convexe joue le rôle que tient la mesure harmonique dans la théorie du problème de Dirichlet classique.

6.f. On peut étudier dans le même esprit la dépendance de la solution $u$ du Problème 6.a à l'égard de $f$, ou même la dépendance simultanée de $u$ à l'égard des données $u^{*}$ et $f$, le couple $\left(u^{*}, f\right)$ étant un élément de l'espace vectoriel $\mathfrak{C}\left(\Omega^{*}\right) \times \mathfrak{C}(\Omega)$.

On note d'abord que l'ensemble $\mathrm{D}$ des $\left(u^{*}, f\right)$ vérifiant la condition de possibilité (6.1) est une partie conique convexe de cet espace vectoriel. Il est clair en effet que $\left(u^{*}, f\right) \in \mathrm{D}$ entraine $\left(\lambda u^{*}, \lambda f\right) \in \mathrm{D}$, quelle que soit la constante $\lambda>0$. 
De plus, si $\left(u_{1}^{*}, f_{1}\right)$ et $\left(u_{2}^{*}, f_{2}\right)$ sont deux éléments de $\mathrm{D}$, on a, pour chaque $a \in \Omega^{*}$,

$$
\begin{aligned}
& \lim _{x \rightarrow a} \sup \left[f_{1}(x)+f_{2}(x)\right] \leqslant \lim _{x \rightarrow a} \sup f_{1}(x)+\lim _{x \rightarrow a} \text { sup } f_{2}(x) \\
& \leqslant u_{\mathbf{1}}^{*}(a)+u_{\mathbf{2}}^{*}(a),
\end{aligned}
$$

c'est-à-dire que la somme $\left(u_{1}^{*}+u_{2}^{*}, f_{1}+f_{2}\right)$ appartient à D.

Pour chaque $x_{0} \in \Omega$, l'application $\left(u^{*}, f\right) \longmapsto u\left(x_{0}\right)$ est convexe de $\mathrm{D}$ dans $\mathbf{R}$. En effet, si $u_{1}$ et $u_{2}$ sont les solutions correspondant respectivement aux données $\left(u_{1}^{*}, f_{1}\right)$ et $\left(u_{2}^{*}, f_{2}\right)$, la fonction $\alpha_{1} u_{1}+\alpha_{2} u_{2}$ (avec $\alpha_{1}$ et $\alpha_{2}$ constantes dans $] 0,1\left[\right.$ telles que $\alpha_{1}+\alpha_{2}=1$ ) est une majorante surharmonique de $\alpha_{1} f_{1}+\alpha_{2} f_{2}$, continue dans $\hat{\Omega}$ et admettant $\alpha_{1} u_{1}^{*}+\alpha_{2} u_{2}^{*}$ comme restriction à la frontière; la Proposition $6 . b$ fournit alors l'inégalité de convexité annoncée.

Du point de vue topologique, enfin, on pourra poser la notation

$$
\left\|\left(u^{*}, f\right)\right\|=\max \left\{\max _{a \in \Omega^{*}}\left|u^{*}(x)\right|, \sup _{b \in \Omega}|f(b)|\right\}
$$

(non nécessairement fini car $\Omega$ n'est pas compact); on constate alors

$$
\left|u_{1}\left(x_{0}\right)-u_{2}\left(x_{0}\right)\right| \leqslant\left\|\left(u_{1}^{*}-u_{2}^{*}, f_{1}-f_{2}\right)\right\| .
$$

En effet, soit $m$ un majorant (fini) du second membre de cette inégalité; cela veut dire

$$
\begin{array}{lll}
u_{2}^{*}-m \leqslant u_{1}^{*} \leqslant u_{2}^{*}+m & \text { dans } & \Omega^{*} \\
f_{2}-m \leqslant f_{1} \leqslant f_{2}+m & \text { dans } & \Omega .
\end{array}
$$

Or $u_{2}+m$ est minimale de $f_{2}+m$, continue dans $\hat{\Omega}$, admettant $u_{2}^{*}+m$ comme restriction à $\Omega^{*}$. Puisque $u_{2}^{*}+m$ majore $u_{1}^{*}$ et que $f_{2}+m$ majore $f_{1}$, les Corollaires 1 et 2 du $\S 6 . b$ montrent que $u_{2}+m$ majore $u_{1}$. Un raisonnement semblable montre que $u_{2}-m$ minore $u_{1}$ donc

$$
\left|u_{1}\left(x_{0}\right)-u_{2}\left(x_{0}\right)\right| \leqslant m
$$

\section{Familles de minimales.}

7.a. Proposition. - Soit do un ensemble de minimales de la fonction continue $f$ dans l'ousert $\Omega$. Si en un point $x_{0} \in \Omega$, 
toutes les fonctions de do ont leurs valeurs majorées par un réel $\mathrm{M}$, il existe un voisinage du point $x_{0}$ sur lequel cet ensemble de fonctions est uniformément équicontinu.

Démonstration. - Soit B une boule de centre $x_{0}$, d'adhérence $\overline{\mathrm{B}}$ contenue dans $\Omega$. Pour toute minimale $u$ de $f$, on notera $u_{\mathrm{B}}$ la fonction continue dans $\overline{\mathrm{B}}$, harmonique dans $\mathrm{B}$, ayant la même restriction que $u$ à la sphère $\partial \mathrm{B}$ (on la construit par l'intégrale de Poisson). On notera de plus

$$
u_{\mathrm{B}}^{\prime}=u-u_{\mathrm{B}}
$$

c'est une fonction $\geqslant 0$ dans $\mathrm{B}$, puisque $u$ est surharmonique.

Soit $p$ la plus petite minimale de $f$; la différence $u-p$ est sousharmonique (cf. Proposition 4.d), donc la différence $u_{\mathrm{B}}^{\prime}-p_{\mathrm{B}}^{\prime}$ est sousharmonique dans $\mathrm{B}$, continue dans $\overline{\mathrm{B}}$ et nulle au bord, par suite $\leqslant 0$ dans $\bar{B}$. Bref :

$$
0 \leqslant u_{\mathbf{B}}^{\prime} \leqslant p_{\mathbf{B}}^{\prime}
$$

En outre, le principe du minimum pour la fonction harmonique $p_{\mathrm{B}}$, puis la continuité de $p$, montrent qu'on peut, en choisissant la boule $B$ assez petite, rendre la fonction $p_{B}^{\prime}$ uniformément petite dans $\mathrm{B}$, donc uniformément petites toutes les $u_{\mathrm{B}}^{\prime}$.

D'autre part $u \geqslant p$, donc $u_{\mathrm{B}} \geqslant p_{\mathrm{B}} ;$ les différences $u_{\mathrm{B}}-p_{\mathrm{B}}$ constituent une famille de fonctions harmoniques $\geqslant 0$ dans $\mathrm{B}$ et l'on a, si $u \in \mathfrak{b}$,

$$
u_{\mathbf{B}}\left(x_{0}\right)-p_{\mathrm{B}}\left(x_{0}\right) \leqslant u\left(x_{0}\right)-p_{\mathbf{B}}\left(x_{0}\right) \leqslant \mathrm{M}-p_{\mathrm{B}}\left(x_{0}\right) .
$$

On en tire classiquement, par les inégalités de Harnack, que cette famille de fonctions harmoniques est équicontinue au point $x_{0}$, donc aussi bien la famille des $u_{\mathrm{B}}$.

Soit alors $\varepsilon>0$; on écrit, pour tout $x \in \mathrm{B}$,

$$
\left|u(x)-u\left(x_{0}\right)\right| \leqslant\left|u_{\mathbf{B}}(x)-u_{\mathrm{B}}\left(x_{0}\right)\right|+\left|u_{\mathrm{B}}^{\prime}(x)\right|+\left|u_{\mathrm{B}}^{\prime}\left(x_{0}\right)\right| .
$$

Choisissons la boule $\mathrm{B}$ assez petite pour que la fonction $p_{\mathrm{B}}^{\prime}$, donc $u_{\mathrm{B}}^{\prime}$, soit moindre que $\frac{\varepsilon}{3}$ dans B. L'équicontinuité de la famille $u_{\mathbf{B}}$ permet de trouver $\eta>0$ (moindre que le 
rayon de B) tel que $\left|x-x_{0}\right| \leqslant \eta$ entraînera

$$
\left|u_{\mathbf{B}}(x)-u_{\mathbf{B}}\left(x_{0}\right)\right| \leqslant \frac{\varepsilon}{3}
$$

quelle que soit $u \in \mathfrak{d}$, et par suite,

$$
\left|u(x)-u\left(x_{0}\right)\right| \leqslant \varepsilon,
$$

ce qui prouve l'équicontinuité des fonctions $u \in A_{\ell}$ au point $x_{0}$.

Cette équicontinuité montre que la propriété de majoration par une constante finie a lieu sur un voisinage compact $K$ de $x_{0}$, d'où équicontinuité simple en chaque point de ce compact. De cette équicontinuité simple, on passe par des arguments classiques, à l'équicontinuité uniforme dans le compact $\mathrm{K}$.

7.b. On tire d'abord ce qui précède :

Proposition. - Soit do une famille (non vide) de minimales de $f$. L'enseloppe inférieure de cette famille est une fonction partout finie et continue. Dans chaque composante connexe de $\Omega$, l'enveloppe supérieure de la famille to est soit partout finie et continue, soit partout égale $\grave{a}+\infty$.

En ce qui concerne l'enveloppe inférieure, elle est en effet partout finie, puisque minorée par $f$ (et même par $p$ ); soit $\mathrm{V}$ un voisinage compact d'un point $x_{0} \in \Omega$ et soit $u_{0} \in \mathcal{A}$. Pour construire dans V l'enveloppe inférieure des $u \in \mathcal{A}_{\mathrm{b}}$ on peut retenir seulement celles de ces fonctions qui sont majorées dans V par $\max u_{0}(\mathrm{~V})$; d'après la Proposition 7.a, cette sousfamille est équicontinue au point $x_{0}$, d'où la continuité de l'enveloppe inférieure.

En ce qui concerne l'enveloppe supérieure, la même Proposition montre immédiatement que si, en un point $x_{0}$, cette enveloppe est finie, elle est continue en ce point, donc encore finie au voisinage: l'ensemble de ces points est donc ouvert. Il reste à montrer que l'ensemble des points où l'enveloppe supérieure vaut $+\infty$ est ouvert également. Soit $x_{0}$ un tel point et $\mathrm{B}$ une boule de centre $x_{0}$, d'adhérence $\overline{\mathrm{B}} \subset \Omega$. Avec les notations du $\S 7 . a$, on voit que l'enveloppe supérieure des fonctions harmoniques positives $u_{\mathrm{B}}-p_{\mathrm{B}}$ vaut $+\infty$ au 
point $x_{0}$ : on déduit classiquement des inégalités de Harnack que cette enveloppe supérieure vaut $+\infty$ partout dans $B$. Il en est donc de même de l'enveloppe supérieure des $u \geqslant u_{\mathbf{B}}$.

7.c. On en vient maintenant à la convergence des suites généralisées de minimales (en particulier, celle des suites ordinaires).

Proposition. - Soit (I, 々) un ensemble d'indices ordonné filtrant à droite. Soit $\left(u_{i}\right)_{i \in I}$ une suite généralisée de minimales de $f$ simplement convergente dans l'ouvert connexe $\Omega$. Alors la fonction limite est soit la constante $+\infty$, soit une minimale de $f$ et la convergence est uniforme sur tout compact $\mathrm{K} \subset \Omega$ au sens de la structure uniforme de $\overline{\mathbf{R}}$. Dans le premier cas (convergence vers $+\infty$ ) cela signifie

$$
\forall r \in \mathbf{R}, \quad \exists i_{r} \in \mathrm{I}:\left(i_{r} \prec i \Longrightarrow u_{i}(\mathrm{~K}) \geqslant r\right) ;
$$

dans le second cas, la convergence est aussi bien uniforme au sens de la structure uniforme additive (métrique) de $\mathbf{R}$.

Démonstration. - Montrons que l'ensemble des $x \in \Omega$ tels que $\lim u_{i}(x)<+\infty$ est ouvert: soit $x_{0}$ dans cet ensemble. Quitte à remplacer I par $J=\left\{i \in \mathrm{I}: i_{0} \prec i\right\}$, pour un $i_{0}$ convenable, ce qui ne modifie pas les convergences, on voit que l'ensemble des $u_{i}\left(x_{0}\right)$ est majoré; la Proposition 7.a montre alors l'équicontinuité des fonctions $u_{i}$ en ce point, de sorte que la limite est encore finie sur un voisinage de $x_{0}$. L'ensemble des $x \in \Omega$ tels que $\lim u_{i}(x)=+\infty$ est ouvert aussi : en effet, si $\lim u_{i}\left(x_{0}\right)=+\infty$, on a, pour une boule B de centre $x_{0}$, avec les notations $d u \S 7 . a$,

$$
\lim \left[u_{i \mathbf{B}}\left(x_{0}\right)-p_{\mathbf{B}}\left(x_{0}\right)\right]=+\infty \text {. }
$$

Comme les fonctions $u_{i \mathrm{~B}}-p_{\mathrm{B}}$ sont harmoniques $\geqslant 0$ dans $B$, il en résulte classiquement, par les inégalités de Harnack, que la limite est $+\infty$ partout dans B, cela, d'ailleurs, uniformément dans une boule contenue et, en fin de compte, uniformément sur le compact $\mathrm{K}$ (moyennant un recouvrement fini par de telles boules).

Le fait que $\Omega$ est connexe implique donc l'alternative de l'énoncé. 
Si la limite est partout finie dans $\Omega$, on trouve, d'après la Proposition 7.a, pour tout $x_{0} \in \Omega$, une boule fermée $\overline{\mathrm{B}} \subset \Omega$ de centre $x_{0}$ sur laquelle la famille des $u_{i}$ est uniformément équicontinue pour la structure uniforme métrique de $\mathbf{R}$ : on en tire la convergence uniforme de la suite généralisée sur cette boule. En appliquant l'inégalité (6.4) au domaine B, on voit que la limite $u$ coïncide nécessairement avec la solution du Problème 6.a pour ce domaine sphérique et la donnée frontière $u^{*}$ (restriction de $u$ à la sphère $\partial \mathrm{B}$ ). Donc $u$ est une minimale de $f$ dans $\mathrm{B}$ et, de même dans toutes les boules d'un recouvrement de $\Omega$. La convergence est, en outre, uniforme, dans un compact $\mathrm{K} \subset \Omega$, puisque $\mathrm{K}$ pourra être recouvert par une famille finie de ces boules.

Corollaire. - L'enveloppe inférieure d'une famille filtrante décroissante de minimales de $f$ dans un ouvert quelconque est une minimale de $f$. L'enseloppe supérieure d'une famille filtrante croissante de minimales de $f$ dans un ouvert connexe est soit la constante $+\infty$, soit une minimale de $f$.

En effet ces familles filtrantes peuvent être considérées comme des suites généralisées dont l'ensemble d'indices est la famille elle-même (avec son ordre numérique naturel ou l'ordre opposé): l'enveloppe supérieure (resp. inférieure) coïncide avec la limite simple de cette suite généralisée.

7.d. L'équicontinuité formulée en 7.a implique, par le théorème d'Ascoli, des propriétés de compacité analogues au théorème de Montel de la théorie des fonctions harmoniques.

Un énoncé synthétique est le suivant:

Proposition. - On suppose pour simplifier $\Omega$ connexe. Dans l'espace fonctionnel $\mathfrak{C}(\Omega, \overline{\mathbf{R}})$ des applications continues de $\Omega$ dans $\overline{\mathbf{R}}$, muni de la topologie de la convergence compacte, la partie A constituée des minimales de $f$ et de la constante $+\infty$ est compacte.

En effet, A est fermé dans l'espace en question, d'après la Proposition 7.c. Comme les fonctions de A prennent leurs valeurs dans l'espace compact $\overline{\mathbf{R}}$, il suffit pour invoquer le théorème d'Ascoli, de montrer l'équicontinuité des $u \in \mathrm{A}$ en chaque point $x_{0} \in \Omega$, pour la structure uniforme de $\overline{\mathbf{R}}$. 
En fait, sur un voisinage compact de $x_{0}$, les fonctions de A prennent leurs valeurs dans l'intervalle $[\mu,+\infty]$, où $\mu \in \mathbf{R}$ est un minorant strict de $f$ sur ce voisinage. D'après la définition de la structure uniforme de $\overline{\mathbf{R}}$ (structure uniforme canonique de cet espace compact; les entourages sont les voisinages de la diagonale dans l'espace compact $\overline{\mathbf{R}}^{2}$ ), on peut fonder la démonstration de l'équicontinuité sur la considération d'entourages $\mathrm{V}$ de la forme suivante; on prend $\varepsilon>0$ (petit) et $r>\max \{\mu, 0\}$ (grand); V, ouvert de $\overline{\mathbf{R}^{2}}$, est la réunion de l'ouvert $\left.\left.\mathrm{V}_{1}=\right] r, \infty\right]^{2}$ et de l'ouvert

$$
\mathrm{V}_{2}=\{(x, y) \in] \mu, 5 r[2:|x-y|<\varepsilon\} .
$$

Pour chacun de ces $\mathrm{V}$, on partage $\mathrm{A}$ en la réunion des deux ensembles

$$
\begin{aligned}
& \mathrm{A}_{1}=\left\{u \in \mathrm{A}: u\left(x_{0}\right)>4 r\right\}, \\
& \mathrm{A}_{2}=\left\{u \in \mathrm{A}: u\left(x_{0}\right) \leqslant 4 r\right\} .
\end{aligned}
$$

D'après la Proposition 7.a, l'ensemble $\mathrm{A}_{2}$ est équicontinu au point $x_{0}$, au sens de la structure uniforme additive de $\mathbf{R}$; il existe donc un voisinage $\beta_{2}$ de $x_{0}$ dans $\Omega$ tel que

$$
\forall u \in \mathrm{A}_{2}, \quad \forall x \in \beta_{2}: \quad\left(u\left(x_{0}\right), u(x)\right) \in \mathrm{V}_{2} .
$$

En ce qui concerne les fonctions de $A_{1}$, on considère comme au $\S 7 . a$, une boule $\overline{\mathrm{B}} \subset \Omega$, de centre $x_{0} ;$ chaque $u \in \mathrm{A}$, (à l'exception de la constante $+\infty$ qui ne pose pas de problème) est décomposée sous la forme

$$
u=u_{\mathbf{B}}-p_{\mathbf{B}}+u_{\mathbf{B}}^{\prime}+p_{\mathrm{B}} .
$$

En se limitant à considérer des $r$ suffisamment grands, on peut, vu (7.1), choisir la boule $\bar{B}$ telle que, partout dans cette boule, on ait

$$
r>p_{\mathbf{B}}^{\prime}+p_{\mathbf{B}} \geqslant u_{\mathbf{B}}^{\prime}+p_{\mathbf{B}} \geqslant p_{\mathbf{B}}>-r
$$

Alors, pour tous les $u \in \mathrm{A}_{1}$, les fonctions harmoniques positives $u_{\mathrm{B}}-p_{\mathrm{B}}$ surpassent $3 r$ au point $x_{0}$. En appliquant les inégalités de Harnack à ces fonctions harmoniques positives, on construit une boule $\bar{\beta}_{1} \subset \mathrm{B}$, de centre $x_{0}$, telle que

$$
u_{\mathrm{B}}-p_{\mathrm{B}} \geqslant 2 r \quad \text { dans } \quad \bar{\beta}_{1} \text {. }
$$


$\mathrm{Vu}$ (7.2) et (7.3), on en tire $u>r$ dans l'intérieur $\beta_{1}$; donc, pour $u \in \mathrm{A}_{1}$,

$$
\forall x \in \beta_{1}: \quad\left(u\left(x_{0}\right), u(x)\right) \in \mathrm{V}_{1} .
$$

Bref, pour $u \in \mathrm{A}$,

$$
\forall x \in \beta_{1} \cap \beta_{2}: \quad\left(u\left(x_{0}\right), u(x)\right) \in \mathrm{V} .
$$

Cela démontre l'équicontinuité.

Corollaire. - On suppose encore $\Omega$ connexe; soit $x_{0} \in \Omega$. L'ensemble des minimales $u$ de $f$ telles que $u\left(x_{0}\right) \leqslant \mathrm{M}$, avec $\mathbf{M} \in \mathbf{R}$ fixe, (resp. telles que $u\left(x_{0}\right)=\mathbf{M}$ ) est une partie compacte de l'espace vectoriel $\mathfrak{C}(\Omega, \mathbf{R})$ muni de la topologie de la convergence compacte.

Il est clair en effet que cet ensemble est une partie de A fermée dans l'espace $\mathfrak{C}(\Omega, \mathbf{R})$; en outre les $u$ en question prennent leurs valeurs dans $\mathbf{R}$, d'après la Proposition 7.b.

Il est important ici de se rappeler que, $\Omega$ étant localement compact, la topologie de la convergence compacte sur $\mathcal{C}(\Omega, \mathbf{R})$ ne dépend que de la topologie de $\mathbf{R}$ et peut être construite en invoquant dans $\mathbf{R}$ n'importe quelle structure uniforme compatible avec ladite topologie. Aussi bien que la structure uniforme de $\overline{\mathbf{R}}$, invoquée tout à l'heure, on peut donc considérer dans le cas présent la structure uniforme additive de $\mathbf{R}$.

\section{BIBLIOGRAPHIE}

[1] M. Brelot, Minorantes sous harmoniques, extrémales et capacités, J. Math. Pures et Appl., 24 (1945), 1-32.

[2] M. Brelot, Eléments de la théorie classique du potentiel, $3^{\mathrm{e}}$ ed., Centre de Documentation Universitaire, Paris, 1965, 4e édition, 1969.

[3] H. Brezis, G. Stampacchia, Sur la régularité dela solution d'inéquations elliptiques, Bull. Soc. Math. France, 96 (1968), 153-180.

[4] J. F. Durand, Résolution numérique de problèmes aux limites sousharmoniques, Thèse de $3^{\mathrm{e}}$ cycle, Montpellier, 1968.

[5] G. Fichera, Problemi elastostatici con vincoli unilaterali : il problema di Signorini con ambigue condizioni al contorno, Atti Accad. Naz. Lincei, Cl. sci. fis. mat. nat., Memorie, Ser. VIII, Vol. VII, fasc. 5 (1964).

[6] H. Lewy, On a Variational Problem with Inequalities on the Boundary J. Math., Mech., 17 (1968), 861-884. 
[7] H. Lewy, G. Stampacchia, On the regularity of the solution of a variation alinequality, Comm. Pure Appl. Math., 22 (1969) 153-188.

[8] J. L. Lions, G. Stampacchia, Variational inequalities, Comm. Pure Appl. Math. 20 (1967), 493-519.

[9] J. J. Moreau, Proximité et dualité dans un espace hilbertien, Bull. Soc. Math. France, 93 (1965), 273-299.

[10] J. J. Moreau, Convexity and duality, in : E. R. Caianiello (editor), Functional Analysis and Optimization, Academic Press (1966), 145-169.

[11] J. J. Moreau, One-sided constraints in hydrodynamics in : Abadie, J. (editor), Nonlinear programming, North Holland Pub. Co. (1967), 261-279.

[12] J. J. Moreau, Principes extrémaux pour le problème de la naissance de la cavitation, Journ. de Mécanique 5 (1966), 439-470.

[13] J. J. Moreau, Fonctionnelles convexes, seminaire sur les Équations aux dérivées partielles, Collège de France, Paris, 1967 (multigraphié $108 \mathrm{p})$.

[14] W. Prager, Elastic solids of limited compressibility, Proc. 9 th. Int. Congr. Appl. Mech. Bruxelles (1956), Vol. 5, 205-211.

[15] W. Prager, Unilateral constraints in mechanics of continua, Atti del Convegno Lagrangiano, Torino (1964), 181-190.

[16] N. SјöвеRg, Sur les minorantes sousharmoniques d'une fonction donnée, Congrès des Math. Scand., Helsingfors, 1938, p. 309-319.

Manuscrit reçu le 9 juin 1970.

Jean-Jacques Moreau,

Centre de Recherches Mathématiques, Université de Montréal,

Case postale 6128, Montréal, P.Q., Canada

et U.E.R. Mathématiques,

place Eugène-Bataillon, 34-Montpellier, France. 